\title{
La calidad de los servicios que perciben los estudiantes de la Universidad Nacional de Chimborazo de la ciudad de Riobamba - Ecuador
}

\begin{abstract}
RESUMEN
El presente trabajo de investigación ha identificado los factores de riesgo y éxito de la calidad de los servicios educativos de los estudiantes de la Universidad Nacional de Chimborazo, y el impacto de los factores de calidad de los servicios percibidos. Si de los 45 factores se logra mejorar en 12 factores que son: comodidad del aula, comodidad de las oficinas, limpieza de los servicios higiénicos, capacidad de respuesta, rapidez en la atención, atención de la quejas, el estudiante sabe dónde quejarse, conformidad del servicio, información útil, seguridad en el campus y desempeño del docente. De aquí se deriva que existe una alta probabilidad de que los estudiantes estén satisfechos con los servicios y recomienden a la institución.
\end{abstract}

Palabras clave: Calidad, servicio, satisfacción.

THE QUALITY OF THE SERVICES PERCEIVED BY THE STUDENTS OF THE NATIONAL UNIVERSITY of CHIMborazo of THE CITY OF Riobamba ECUADOR

\section{ABSTRACT}

The present research work has identified the risk and success factors of the quality of the educational services of the students of the National University of Chimborazo and the impact of the quality factors of the services perceived. If from the 45 factors we can improve on 12 factors that are: comfort of the classroom, comfort of offices, cleanliness of the toilet, answer's capacity, quickness of care, attention to complaints, student knows where to complain, useful information, campus safety, and teacher performance. It follows that there is a high probability that students will be satisfied with the services and recommend to the institution.

Keywords: Quality, service, satisfaction.

\section{INTRODUCCIÓN}

La calidad educativa es un concepto bastante impreciso y controvertido en la investigación existe una política de la discusión. Para diferentes personas, la definición puede variar por lo que los indicadores que se utilizan para describir la calidad de la educación pueden ser diferentes (Fuller, 1986), algunos pueden hacer hincapié en la calidad de los insumos para los sistemas de educación, mientras que otros hacen hincapié en la calidad del proceso y los resultados. Si se refiere a insumos, procesos, resultados, o todos ellos, la definición de la calidad de la educación a menudo se puede asociar a la aptitud para el uso, la satisfacción de las necesidades de los grupos estratégicos (por ejemplo, los responsables políticos, padres de familia, profesores y estudiantes, etc.) (Cheng, 1997).

El préstamo de las ideas de enfoque total del sistema de gestión y calidad, (Cheng, 1995), define la calidad de la educación de la siguiente manera: Calidad de la educación es el carácter del conjunto de elementos de la entrada, proceso y salida del sistema educativo que ofrece servicios que satisfacen por completo tanto grupos estratégicos internos y externos mediante el cumplimiento de sus expectativas explícitas e implícitas.

La aceptación de esta definición, el concepto de calidad de la educación implicará las características del insumo, proceso, producto y múltiples grupos de una institución educativa. Por lo tanto, por otra parte, las expectativas de los diferentes, cuando no contradictorias. A menudo es difícil para una institución educativa para satisfacer todas las expectativas o las necesidades a la vez. Por lo tanto, no es raro que la calidad de la educación en una institución que se percibe elevado a algunos aspectos de la educación puede ser de alta calidad, pero otros aspectos pueden ser de baja calidad (Hughes, 1998).

En otras palabras, a partir de las diferentes concepciones de la calidad de educación existen diferentes inquietudes acerca de la consecución de la calidad de la educación, varias personas pueden usar diferentes indicadores para evaluar la calidad de la educación y las diferentes estrategias para lograr la calidad de la educación. El enfoque de estos indicadores y estrategias no puede incluir necesariamente todos los aspectos de la entrada,

\footnotetext{
Docente de la Universidad Nacional de Chimborazo, Master en Finanzas.

E-mail:wsaltos@unach.edu.ec

2 Docente de la Escuela Superior Politécnica de Chimborazo, Doctor en Gestión de Empresas. E-mail: stalin.arguello@spoch.edu.ec
} 
proceso y resultado de una institución educativa. (Cheng \& Tam, 1997).

La revisión de la literatura reveló dos estudios con el uso de SERVQUAL en la educación superior. (Boulding \& Kalra \& Staeling \& Zeithanml, 1992), utilizaron SERVQUAL en un estudio para probar un modelo de proceso de la calidad del servicio. El equipo de investigación utilizó un SERVQUAL modificada de 36 ítems para capturar las expectativas y las percepciones asociadas a un servicio educativo.

El formato de las expectativas de SERVQUAL por aproximadamente la mitad de la muestra de 177 fue en la forma de lo que el cliente va a suceder espera, la otra mitad de lo que debería ocurrir en el proceso educativo. También solicitaron información en cuanto a la probabilidad de que estos estudiantes serían recomendar esta escuela o para contribuir con dinero en el futuro.

(Edwards, 1991), afirma enfáticamente que el concepto de calidad es un significante y no un significado. El reclamo por la ambigüedad del término surge porque se expresa que él está definido por un solo significado. Sin embargo, el concepto de calidad como el de bueno, o la muerte son significantes que pueden adquirir múltiples significados. Entonces el concepto de calidad es de por sí ambiguo.

Para Edwards, esa calidad debe definirse en cada situación y los significados dependerán de la perspectiva social desde la que se hacen; de los sujetos que la enuncian (profesores, padres, agencia de planificación) y desde el lugar en que se hace (aula, ministerio, etc.). Por lo mismo, el concepto de calidad, en fin, conlleva posicionamiento político, social y cultural frente a lo educativo, (Edwards, 1991) en su concepto de la calidad de la Educación.

La calidad es un factor estratégico que determina el éxito a largo plazo de una organización. Calidad tiene que ver con la calidad requerida de gestión, la calidad humana, los bienes y la calidad del servicio con el fin de satisfacer las necesidades del consumidor (Keskin, 1998). La calidad no es sólo un cumplimiento de ciertas características, sino también el cumplimiento de las necesidades del consumidor (Sekerkaya, 1997). La definición de la calidad varía de persona a persona, y el consumidor decide si la calidad del servicio se cumple o no, cuando se ha beneficiado del servicio.

(Parasuraman \& Zeithaml \& Berry, 1988), definen la calidad del servicio como "un juicio global o actitud en relación con la excelencia en general o la superioridad del servicio" (p.22). y conceptualizan la evaluación de un cliente de la calidad general del servicio mediante la aplicación (1980) modelo de desconfirmación de Oliver, la brecha entre las expectativas y la percepción (modelo de la brecha) de los niveles de rendimiento del servicio. Además, proponen que el rendimiento global de la calidad de servicio puede ser determinado por una escala de medida llamada "SERVQUAL", que utiliza cinco dimensiones genéricas: Tangibles, Fiabilidad, Capacidad de respuesta, Seguridad, Empatía.

La calidad en la educación es un concepto polivalente que depende del actor que utilicemos como referencia. (Scharager \& Aravena, 2010), señalan que para unos implica empleabilidad, desarrollo de competencias o saberes; mientras que para otros se asocia al desarrollo humano y a las capacidades en ámbitos que van más allá del sector productivo.

(González \& Torre, 2007), señalan que se puede evaluar mediante indicadores, criterios y variables que permitan garantizar a la sociedad la pertinencia de los saberes ofertados por las ÍES, los cuales se concentran tradicionalmente en rubros tales como los planes y programas de estudio, la plantilla docente, los alumnos, la infraestructura, el financiamiento y los procesos académico-administrativos (Gil, 2006).

El propósito del trabajo investigativo es establecer los niveles de calidad de los servicios que brinda la Universidad Nacional de Chimborazo de la ciudad de Riobamba-Ecuador.

\section{METODOLOGÍA}

Según (Hernández \& Fernandez \& Baptista, 2010), en su libro Metodología de la investigación quinta edición, el tipo de investigación es descriptiva, y explicativa.

Es descriptiva, porque se hace un diagnóstico de las variables de estudio. Explicativa, porque se identifica los servicios que brindan a los estudiantes.

El diseño de investigación es no experimental y de corte transversal, según, (Hernández \& Fernandez \& Baptista, 2010), Organismos y Procesos de Certificación de la Calidad de la Educación Superior Privada en México. Los diseños transeccionales o transversales se trata de descripciones, pero no de categorías, conceptos, objetos ni variables individuales, sino de sus relaciones, sean áreas puramente correlaciónales o relaciones causales.

En estos diseños lo que se mide-analiza (enfoque cuantitativo), o evalúa-analiza (enfoque cualitativo) es la asociación entre categorías, conceptos, objetos o variables en un tiempo determinado, a veces únicamente en términos correlaciónales, otras en 
términos de relación causa-efecto, pero siempre en un momento específico.

Es no experimental porque no manipula las variables de estudio. Es de corte transversal porque levanta la información por única vez. Es cuantitativa porque mide las variables de estudio.

Dónde:

\section{X: Calidad de los Servicios}

Dimensiones de la calidad de los servicios según el modelo SERVQUAL que son cinco que se define así:

X1: Elementos tangibles, X2: Fiabilidad, X3: Capacidad de respuesta, X4: Empatía, X5: Seguridad.

El presente estudio tiene como unidad de análisis a los estudiantes de la Universidad Nacional del Chimborazo de tal forma que reúnan las condiciones para evaluar los servicios educativos y luego realizar un diagnóstico de la calidad de los servicios que brinda a los estudiantes.

La población está conformada por todas las carreras profesionales de la Universidad Nacional del Chimborazo y el instituto de posgrado, cuya totalidad es de 8377 estudiantes.

La técnica de muestreo fue por conveniencia, ya que existen estudiantes que no disponen de tiempo para responder el cuestionario.

Para el tamaño de muestra se utilizó la fórmula de:

$$
n=\frac{Z_{\alpha}^{2} \cdot N \cdot p \cdot q}{i^{2}(N-1)+Z_{\alpha}^{2} \cdot p \cdot q}
$$

Donde:

$\mathrm{n}=$ Tamaño de la muestra

$\mathrm{N}=$ Tamaño de la población

$Z_{\infty}=1.96$ al cuadrado (si la seguridad es del 95\%)

$p=$ proporción esperada (en este caso 0.5 )

$q=1-p($ en este caso 1-0,5 =0.5)

$i=\operatorname{error}(5 \%)$

\section{Semestral}

$n=\frac{(1.96)^{2} *(5290) *(0.5) *(0.5)}{(0.0025) *(5290-1)+(1962) *(0.5) *(0.5)} \quad n=335$

Anual

$n=\frac{(1.96)^{2} *(2225) *(0.5) *(0.5)}{(0.0025) *(2225-1)+(1962) *(0.5) *(0.5)} \quad n=259$
UFAP

$n=\frac{(1.96)^{2} *(381) *(0.5) *(0.5)}{(0.0025) *(381-1)+(1962) *(0.5) *(0.5)} \quad n=77$

Posgrado

$n=\frac{(1.96)^{2} *(481) *(0.5) *(0.5)}{(0.0025) *(841-1)+(1962) *(0.5) *(0.5)} \quad n=148$

Muestra total 819 estudiantes.

\section{RESULTADOS}

\section{ELEMENTOS TANGIBLES}

En esta sección se presenta la descripción y el análisis de los niveles de las dimensiones de la calidad de los servicios (SERVQUAL), para cada una de las dimensiones.

\section{Dimensión 1.- Elementos Tangibles}

En la tabla 1 se determina la calidad de los servicios ofertados por la Universidad Nacional de Chimborazo, mediante los elementos tangibles.

Tabla 1. Distribución de los niveles de los elementos tangibles la calidad del servicio de los estudiantes de la Universidad Nacional Chimborazo Riobamba - Ecuador.

\begin{tabular}{lrrrr}
\hline & Frecuencia & Porcentaje & $\begin{array}{c}\text { Porcentaje } \\
\text { válido }\end{array}$ & $\begin{array}{r}\text { Porcentaje } \\
\text { acumulado }\end{array}$ \\
\hline Deficiente & 7 & .9 & .9 & .9 \\
Mala & 38 & 4.6 & 4.6 & 5.5 \\
Regular & 143 & 17.5 & 17.5 & 23.0 \\
Buena & 448 & 54.7 & 54.7 & 77.7 \\
Excelente & 183 & 22.3 & 22.3 & 100.0 \\
Total & 819 & 100.0 & & \\
\hline
\end{tabular}

Fuente: Elaboración propia.

Se observa que el $54.7 \%$ de los alumnos de la Universidad Nacional de Chimborazo Riobamba Ecuador, mencionan que los elementos tangibles son bueno; es decir, la limpieza, el ambiente, decoración, la limpieza en los servicios higiénicos, la infraestructura, la comodidad, la apariencia y presentación del personal, la facilidad de ubicación del local. Mientras que el $22.3 \%$ de los alumnos de la Universidad Nacional de Chimborazo Riobamba 
Ecuador menciona que los elementos tangibles son excelentes, y solo $0.9 \%$ de los alumnos menciona que los elementos tangibles son percibidos como deficientes.

\section{Dimensión 2.- Capacidad de Respuesta}

En la tabla 2 se establece la calidad de los servicios ofertados por la Universidad Nacional de Chimborazo, mediante la capacidad de respuesta.

Tabla 2. Distribución de los niveles de la dimensión de la capacidad de respuesta de la calidad del servicio de los estudiantes de la Universidad Nacional Chimborazo Riobamba - Ecuador.

\begin{tabular}{lcccc}
\hline & Frecuencia & Porcentaje & $\begin{array}{c}\text { Porcentaje } \\
\text { válido }\end{array}$ & $\begin{array}{c}\text { Porcentaje } \\
\text { acumulado }\end{array}$ \\
\hline Deficiente & 14 & 1.7 & 1.7 & 1.7 \\
Mala & 71 & 8.7 & 8.7 & 10.4 \\
Regular & 229 & 28.0 & 28.0 & 38.3 \\
Buena & 380 & 46.4 & 46.4 & 84.7 \\
Excelente & 125 & 15.3 & 15.3 & 100.0 \\
\hline Total & 819 & 100.0 & 100.0 & \\
\hline
\end{tabular}

Fuente. Elaboración propia.

Se observa que el $46.4 \%$ de los estudiantes de la Universidad Nacional de Chimborazo Riobamba Ecuador, menciona que la capacidad de respuesta ante las solicitudes de los estudiantes es regular; es decir, la puntualidad en la entrega de documentos es de calidad regular, la disponibilidad de ayuda con prontitud por parte del personal, la información recibida no siempre es suficiente y satisfactoria es decir algunas veces permite absolver las dudas e inquietudes de los alumnos, la exactitud de respuesta que le dieron ante su petición algunas veces fue satisfactoria, el horario de atención no siempre se acomoda a su disponibilidad de tiempo, si los alumnos desean presentar alguna queja existe un grado de desorientación de cómo y dónde debe hacerlo.

Mientras que el $15.3 \%$ de los alumnos de la Universidad Nacional de Chimborazo Riobamba Ecuador, menciona que es excelente, y solo $1.7 \%$ manifiestan que es deficiente.

\section{Dimensión 3.- Empatía}

En la tabla 3 se determina la calidad de los servicios ofertados por la Universidad Nacional de Chimborazo, mediante la empatía.
Tabla 3. Distribución de los niveles de empatía de la calidad del servicio de los estudiantes de la Universidad Nacional Chimborazo Riobamba - Ecuador.

\begin{tabular}{lrrrr}
\hline & Frecuencia & Porcentaje & $\begin{array}{c}\text { Porcentaje } \\
\text { válido }\end{array}$ & $\begin{array}{r}\text { Porcentaje } \\
\text { acumulado }\end{array}$ \\
\hline Deficiente & 16 & 2.0 & 2.0 & 2.0 \\
Mala & 63 & 7.7 & 7.7 & 9.6 \\
Regular & 180 & 22.0 & 22.0 & 31.6 \\
Buena & 406 & 49.6 & 49.6 & 81.2 \\
Excelente & 154 & 18.8 & 18.8 & 100.0 \\
\hline \multicolumn{1}{r}{ Total } & 819 & 100.0 & 100.0 & \\
\hline
\end{tabular}

Fuente. Elaboración propia.

Se observa que el $49.6 \%$ de los alumnos de la Universidad Nacional de Chimborazo Riobamba Ecuador expresan que la empatía que muestra el personal de universidad es buena; es decir, el trato personalizado por parte de los empleados es buena, además el empeño del personal por escuchar con atención la necesidad de cada alumno, asimismo el uso del lenguaje por parte del personal es sencillo, tienen buena la comprensión respecto a la necesidad que tiene cada alumno, la atención de quejas presentadas formalmente es atendido y la orientación que se da cada alumno es percibida como buena.

Mientras que el $22.0 \%$ de los estudiantes de la Universidad Nacional de Chimborazo Riobamba Ecuador, menciona que la empatía del personal es percibida como regular, y solo el $2.0 \%$ de los mencionan que la empatía de la es percibida como deficiente.

\section{Dimensión 4.- Fiabilidad}

En la tabla 4se establece la calidad de los servicios ofertados por la Universidad Nacional de Chimborazo, mediante la fiabilidad.

Tabla 4. Distribución de los niveles de la fiabilidad de la calidad del servicio de los estudiantes de la Universidad Nacional Chimborazo Riobamba - Ecuador.

\begin{tabular}{lrrrr}
\hline & Frecuencia & Porcentaje & $\begin{array}{c}\text { Porcentaje } \\
\text { válido }\end{array}$ & $\begin{array}{r}\text { Porcentaje } \\
\text { acumulado }\end{array}$ \\
\hline Deficiente & 19 & 2.3 & 2.3 & 2.3 \\
Mala & 89 & 10.8 & 10.8 & 13.1 \\
Regular & 214 & 26.1 & 26.2 & 39.2 \\
Buena & 374 & 45.7 & 45.7 & 85.0 \\
Excelente & 123 & 15.0 & 15.0 & 100.0 \\
\hline \multicolumn{1}{c}{ Total } & 819 & 100.0 & 100.0 & \\
\hline
\end{tabular}

Fuente. Elaboración propia. 
Se observa el $45.7 \%$ de los alumnos de la Universidad Nacional de Chimborazo Riobamba Ecuador, menciona que la fiabilidad del servicio que brinda la universidad es buena; es decir, que la rapidez de la atención es buena, el desempeño de los trabajadores, los afiches y folletos responden a la necesidad de información de manera aceptable, los medios de información del servicio, el número de personas atendiendo respecto a los alumnos que requiere el servicio es suficiente, el uso de tecnología moderna en las clases es la percibida como buena, la facilidad para encontrar la información que necesita en la página web, la temperatura del medio ambiente también es percibida como buena, además implica la habilidad que tiene la Organización para ejecutar el servicio prometido de forma adecuada y constante también es percibida como calidad buena. Mientras que el $26.1 \%$ de los estudiantes de la Universidad Nacional de Chimborazo Riobamba Ecuador, menciona que la fiabilidad es regular, y solo $2.3 \%$ de los alumnos, menciona que la fiabilidad de es deficiente.

\section{Dimensión 5.- Seguridad}

En la tabla 5 se determina la calidad de los servicios ofertados por la Universidad Nacional de Chimborazo, mediante la seguridad.

Tabla 5. Distribución de los niveles de seguridad de la calidad de los servicios de los estudiantes de la Universidad Nacional Chimborazo Riobamba Ecuador.

\begin{tabular}{lrrrr}
\hline & Frecuencia & Porcentaje & $\begin{array}{c}\text { Porcentaje } \\
\text { válido }\end{array}$ & $\begin{array}{r}\text { Porcentaje } \\
\text { acumulado }\end{array}$ \\
\hline Deficiente & 10 & 1.2 & 1.2 & 1.2 \\
Mala & 54 & 6.6 & 6.6 & 7.8 \\
Regular & 140 & 17.1 & 17.1 & 24.9 \\
Buena & 410 & 50.0 & 50.0 & 74.9 \\
Excelente & 205 & 25.0 & 25.1 & 100.0 \\
\hline \multicolumn{1}{r}{ Total } & 819 & 100.0 & & \\
\hline
\end{tabular}

Fuente. Elaboración propia

Se observa el $50.0 \%$ de los alumnos de la Universidad Nacional de Chimborazo Riobamba Ecuador, manifiestan que la seguridad de los servicios que brinda la Universidad es buena; es decir, la conformidad respecto al servicio que se ofrecen a los estudiantes es percibida como buena, la seguridad en las respuestas que se le da es percibida como buena, el personal que atiende en la saluda y se identifica correctamente es percibida como buena, la información que recibe los alumnos es realmente lo que necesita la califican como buena, la seguridad en los ambientes de atención es buena, la sencillez y simplicidad respecto a los tramites es percibida como buena, el clima laboral que se percibe de las oficinas es buena, asimismo la visibilidad e interacción con es personal es percibida como buena.

Mientras que el $3.5 \%$ de los, menciona que la seguridad del servicio es regular, y solo $2.5 \%$ de los, menciona que la seguridad del servicio es excelente.

\section{DESEMPEÑO DOCENTE}

\section{Dimensión 1.- Planificación}

En la tabla 6 se establece el desempeño docente en la universidad nacional de chimborazo, mediante la planificación.

Tabla 6. Distribución los niveles de planificación que realiza el docente y la percepción de los estudiantes de la Universidad Nacional Chimborazo Riobamba - Ecuador.

\begin{tabular}{lrrrr}
\hline & Frecuencia & Porcentaje & $\begin{array}{c}\text { Porcentaje } \\
\text { válido }\end{array}$ & $\begin{array}{r}\text { Porcentaje } \\
\text { acumulado }\end{array}$ \\
\hline Deficiente & 21 & 2.5 & 2.5 & 2.5 \\
Regular & 420 & 51.3 & 51.3 & 53.8 \\
Bueno & 378 & 46.2 & 46.2 & 100.0 \\
Excelente & 0 & 0 & 100.0 & \\
$\quad$ Total & 819 & 100.0 & & \\
\hline
\end{tabular}

Fuente. Elaboración propia.

Se observa que el $51.3 \%$ de los estudiantes de la Universidad Nacional de Chimborazo Riobamba Ecuador, mencionan que en la planificación que realizan los docentes es regular; es decir, la explicación que da el docente con respecto al curso es percibida con una calidad regular, los objetivos del curso que presenta el docente es percibida como regular, la explicación del desarrollo del curso y los detalles del silabo son percibidos como calidad regular. Mientras que el $46.2 \%$ de los estudiantes de la Universidad Nacional de Chimborazo Riobamba Ecuador mencionan que la planificación que realiza el docente es buena, y solo $2.5 \%$ de los estudiantes expresan como deficientes. 


\section{Dimensión 2.- Metodología}

En la tabla 7 se determina el desempeño docente en la Universidad Nacional de Chimborazo, mediante la metodología.

Tabla 7. Distribución de la calidad de la metodología empleada por docente que es percibida por los estudiantes de la Universidad Nacional Chimborazo Riobamba - Ecuador.

\begin{tabular}{lrrrr}
\hline & Frecuencia & Porcentaje & $\begin{array}{c}\text { Porcentaje } \\
\text { válido }\end{array}$ & $\begin{array}{r}\text { Porcentaje } \\
\text { acumulado }\end{array}$ \\
\hline Deficiente & 23 & 2.8 & 2.8 & 2.8 \\
Regular & 384 & 46.9 & 46.9 & 49.7 \\
Bueno & 412 & 50.3 & 50.3 & 100.0 \\
Excelente & 0 & 0 & 0 & 100.0 \\
\hline \multicolumn{1}{r}{ Total } & 819 & 100.0 & 100.0 & \\
\hline
\end{tabular}

Fuente: Elaboración propia.

Se observa que el $50.3 \%$ de los estudiantes de la Universidad Nacional de Chimborazo Riobamba Ecuador, expresan que la metodología empleada por el docente es buena; es decir, la comunicación clara y precisa es percibida como buena, los recursos que utiliza para el desarrollo de sus clases es percibida como buena, y retroalimentación que da el docente en cada clase también es percibida como buena por el $50.3 \%$ de los estudiantes.

Mientras que el $46.9 \%$ de los alumnos de la Universidad Nacional de Chimborazo Riobamba Ecuador perciben que la metodología que emplea el docente es de calidad regular, y solo $2.8 \%$ de los estudiantes mencionan que es deficiente.

\section{Dimensión 3.- Disposición docente}

En la tabla 8 se establece el desempeño docente en la Universidad Nacional de Chimborazo, mediante la disposición docente.

Tabla 8. Distribución del grado de disposición del docente que perciben los estudiantes de la Universidad Nacional Chimborazo Riobamba - Ecuador.

\begin{tabular}{lrrrr}
\hline & Frecuencia & Porcentaje & $\begin{array}{c}\text { Porcentaje } \\
\text { válido }\end{array}$ & $\begin{array}{r}\text { Porcentaje } \\
\text { acumulado }\end{array}$ \\
\hline Deficiente & 19 & 2.3 & 2.3 & 2.3 \\
Regular & 342 & 41.8 & 41.8 & 44.1 \\
Bueno & 457 & 55.8 & 55.8 & 99.9 \\
Excelente & 1 & .1 & .1 & 100.0 \\
\hline \multicolumn{1}{r}{ Total } & 819 & 100.0 & 100.0 & \\
\hline
\end{tabular}

Fuente. Elaboración propia.
Se observa que el $55.8 \%$ de los estudiantes de la Universidad Nacional de Chimborazo Riobamba Ecuador, mencionan que la disposición del docente es buena; es decir, que la amabilidad y el respecto que muestra el docente es percibida como buena, el interés que transmite el docente también es percibida como buena, la forma en que comunica, el interés, y la disposición a responder consultas es percibida como una calidad buena por parte de los estudiantes.

Mientras que el $41.8 \%$ de los estudiantes de la Universidad Nacional de Chimborazo Riobamba Ecuador menciona que la disposición del docente es regular, y solo $2.3 \%$ de los estudiantes mencionan que la disposición que manifiesta el docente es percibida como deficiente.

\section{Dimensión 4.- Calidad de evaluación Docente}

En la tabla 9 se determina el desempeño docente en la Universidad Nacional de Chimborazo, mediante la calidad de evaluación docente.

Tabla 9. Distribución de los niveles de la calidad de evaluación que realiza el docente a los estudiantes de la Universidad Nacional Chimborazo Riobamba - Ecuador

\begin{tabular}{lrrrr}
\hline & Frecuencia & Porcentaje & $\begin{array}{c}\text { Porcentaje } \\
\text { válido }\end{array}$ & $\begin{array}{c}\text { Porcentaje } \\
\text { acumulado }\end{array}$ \\
\hline Deficiente & 9 & 1.1 & 1.1 & 1.1 \\
Regular & 321 & 40.1 & 40.1 & 41.2 \\
Bueno & 481 & 58.7 & 58.8 & 100.0 \\
Excelente & 0 & 0 & 100.0 & \\
\hline \multicolumn{1}{r}{ Total } & 819 & 100.0 & & \\
\hline
\end{tabular}

Fuente. Elaboración propia.

Se observa que el $58.7 \%$ de los estudiantes de la Universidad Nacional de Chimborazo Riobamba Ecuador, mencionan que en general el desempeño docente con sus cuatro dimensiones (planificación, metodología, disposición y evaluación) es percibida como buen desempeño.

Mientras que el $40.1 \%$ de los estudiantes de la Universidad Nacional de Chimborazo Riobamba Ecuador mencionan que el desempeño docente es regular, y solo $1.1 \%$ de los estudiantes mencionan que el desempeño docente es deficiente. 


\section{CONCLUSIONES Y RECOMENDACIONES}

- Se concluye que las dimensiones de la calidad de servicios ofertados por la Institución inciden de manera positiva en los estudiantes.

- En el desempeño Docente (planificación, metodología, disposición y evaluación) existe conformidad de la calidad de los servicios percibida por los estudiantes.

- Se observa que el $54.7 \%$ de los elementos tangibles son buenos; mientras que el $22.3 \%$ son excelente, y solo $0.9 \%$ son deficientes.

- Se observa el $50.0 \%$ es bueno, en seguridad, mientras que el $3.5 \%$ es regular, y solo $2.5 \%$ es excelente.

- Se observa que el $46.4 \%$ menciona que la capacidad de respuesta es regular, el $15.3 \%$ menciona que es excelente, y solo $1.7 \%$ manifiestan que es deficiente.

- Se observa que el $49.6 \%$ expresan que la empatía es buena; mientras que el $22.0 \%$ es percibida como regular, y solo el $2.0 \%$ es percibida como deficiente.

- Se observa el $45.7 \%$ menciona que la fiabilidad del servicio que brinda la universidad es buena; mientras que el $26.1 \%$ es regular, y solo $2.3 \%$ es deficiente.

- Se recomienda controlar y mejorar cada uno de los procesos de acuerdo a los resultados, y cada uno de los factores para lograr calidad en el servicio.

- Se recomienda continuar atendiendo cada uno de los elementos para lograr un mejoramiento continuo.

- Se recomienda presupuestar para contar con los recursos que permitan ejecutar obras de infraestructura para mejorar la calidad de los servicios.

- Se recomienda garantizar seguridad interna en todos los estamentos universitarios y así mejorar el porcentaje y subir a excelente.

- Se recomienda aplicar flujos de procesos para optimizar los tiempos y los recursos en la respuesta a los estudiantes en sus requerimientos.

- Se recomienda atender y preservar la uniformidad de servicios educativos para tener una mayor cobertura y participación en el sector educativo y así lograr mayor competitividad.

- Se recomienda realizar los trámites solicitados cumpliendo las normativas y reglamentos de la institución garantizando así la transparencia en cada uno de los procesos.

\section{REFERENCIAS BIBLIOGRÁFICAS}

[1] Boulding, W., Kalra, A., Staeling, R. \& Zeithaml, A. (1992). A Dynamic Process Model of Service Quality: From Expectations to Behavioral Intentions. Joumal of Marketing Research, 30, 7-27.

[2] Cheng, P. W. (1997). From Covariation to Causation: A Causal Power Theory. Psychological Review, 104, 367- 405.

[3] Cheng, Q. (1995). The Perimeter-area Fractal Model and its Application in Geology. Mathematical Geology, 27, 69-82.

[4] Cheng., \& Tam, W. M. (1997). Multi-models of Quality in Education. Quality Assurance in Education. 5 (1), 22-31.

[5] Edwards, V. (1991). El Concepto de Calidad de la Educación, Quito: Editorial Frónesis.

[6] Fuller, B. (1986). Raicing School Quality in Developing Countries, What Investments Boost Learning. World Bank discussion Pappers 2. Washington D. C., USA : The World Bank.

[7] Gil, H. (2006). La Evaluación, Acreditación en las Instituciones de Educación Superior Públicas de México: Estrategias de Calidad Educativa. En V. Rosario; E. Marúm; R. Vargas; J. Arroyo y V. González (coords.). In Acreditación y Certificación de la Educación Superior: Experiencias, Realidades y Retos para las IES (pp. 423-430). México: Universidad de Guadalajara.

[8] González, L., \& Torre, D. (2007). Acreditación y Fomento de la Calidad. La Experiencia Chilena de las últimas Décadas. En C. Sanyal, y J. Tres (coords.). La Educación Superior en el Mundo 2007: Acreditación para la Garantía de la Calidad: ¿Qué está en juego? (pp. 298306). Madrid: Mundiprensa.

[9] Hernández R., Fernandez C., \& Baptista L. (2010). Metodología de la Investigación. México: Editorial Mc Graw Hill.

[10] Hughes, S. (1998). Getting to the Point: Evolutionary Change in Prehistoric Weaponry. Journal of Archaeological Method and Theory, 5, 345-407.

[11] Keskin, G. (1998). Yasam Kalitesinde Hizmet Kalitesinin Onemi: Belediye Toplu Tasimacilik 
Hizmetleri iizerine Bir Uygulana. Pazarlana Diinyasi, 67, 18-22.

[12] Parasuraman, A., Zeithaml VA., \& Berry, L. L. (1988). SERVQUAL: A Multiple-ltem Scale for Measuring Consumer Perceptions of Service Quality. Journal of Retailing, 16, 12-40.

[13] Scharager, J. \& Aravena, M. T. (2010). Impacto de las Políticas de Aseguramiento de la Cali- dad en Programas de Educación Superior: Un Estudio Exploratorio. Calidad En La Educación, 32, 15-42.

[14] Sekerkaya, A. K. (1997). Bankacilik hizmetlerinde algilanan toplam kalite olcumu. Ankara: Sermaye Piyasası Kurulu (Yayin No:87). 